\title{
Hypertrophic cardiomyopathy: the interrelation of disarray, fibrosis, and small vessel disease
}

\author{
A M Varnava, P M Elliott, S Sharma, W J McKenna, M J Davies
}

\begin{abstract}
Objective-To make a quantitative assessment of the relation between disarray, fibrosis, and small vessel disease in hypertrophic cardiomyopathy.

Design-Detailed macroscopic and histological examination at 19 segments of the left and right ventricle and the left atrial free wall.

Patients-72 patients with hypertrophic cardiomyopathy who had suffered sudden death or progression to end stage cardiac failure (resulting in death or heart transplantation).

Main outcome measures-The presence of scarring, atrial dilatation, and a mitral valve impact lesion were noted, and heart weight, wall thickness, per cent disarray, per cent fibrosis, and per cent small vessel disease quantitated for each heart.

Results-Within an individual heart the magnitude of hypertrophy correlated with the severity of fibrosis $(p=0.006)$ and disarray $(p=0.0002)$. Overall, however, total heart weight related weakly but significantly to fibrosis $(r=0.4, \mathrm{p}=0.0001)$ and small vessel disease $(r=0.3$, $\mathrm{p}=0.03)$, but not to disarray. Disarray was greater in hearts with mild left ventricular hypertrophy (maximum wall thickness $<20 \mathrm{~mm}$ ) and preserved systolic function $(60.9(26) \% v 43$ $(20.4) \%$ respectively, $\mathrm{p}=0.02)$ and hearts without a mitral valve impact lesion $(26.3 \% v 18.9 \%$, $\mathrm{p}=0.04)$, but was uninfluenced by sex. Fibrosis was influenced by sex $(7 \%$ in male patients and $4 \%$ in female, $\mathrm{p}=0.04)$, but not by the presence of an impact lesion. No relation was found between disarray, fibrosis, and small vessel disease.

Conclusions-Myocyte disarray is probably a direct response to functional or structural abnormalities of the mutated sarcomeric protein, while fibrosis and small vessel disease are secondary phenomena unrelated to disarray, but modified by factors such as left ventricular mass, sex, and perhaps local autocrine factors.
\end{abstract}

(Heart 2000;84:476-482)

Keywords: hypertrophic cardiomyopathy; histopathology; small vessel disease

In 1958 Teare described asymmetric left ventricular hypertrophy in several family members who died suddenly. ${ }^{1}$ Since this description, hypertrophic cardiomyopathy is now considered a familial disease characterised morphologically by unexplained hypertrophy, and at the histological level by myocyte disarray, ${ }^{2}$ fibrosis, ${ }^{3}$ and abnormalities of the intramyocardial small vessels. ${ }^{4}$ As the molecular genetics of the condition become unravelled $^{5-7}$ it is increasingly important to understand the basic pathology and the interplay between disarray, fibrosis, and small vessel disease.

To date most published reports on the histopathology of hypertrophic cardiomyopathy have derived from work undertaken during the 1970 s and early 1980 s, ${ }^{389}$ in an era when the broader phenotype of hypertrophic cardiomyopathy was not fully appreciated. It is now increasingly apparent that this is a heterogeneous disease. Morphologically there is not just asymmetrical septal hypertrophy-distal and concentric hypertrophy are now well described, and indeed hypertrophy may be mild or even absent in rare cases. It is also clear that patients with hypertrophic cardiomyopathy may progress to a burnt out phase, characterised by wall thinning, cavity enlargement, and impaired systolic function. ${ }^{10}{ }^{11}$ No study has yet related the histology to these macroscopic findings in a systematic fashion.
In order to clarify these issues we have performed a quantitative assessment of the distribution of disarray, fibrosis, and small vessel changes in a large consecutive group of patients with hypertrophic cardiomyopathy and have evaluated the interrelations between these features and their relation to the macroscopic findings.

\section{Methods}

Seventy two hearts with a diagnosis of hypertrophic cardiomyopathy were obtained at necropsy or transplantation. The diagnosis was based on the typical macroscopic (increased heart weight) and microscopic findings (disarray occupying more than $10 \%$ of the myocardium). ${ }^{2}{ }^{12}$ Nine cases did not fulfil the criteria of increased heart weight, but were included because the degree of disarray was sufficient to be diagnostic. Before death, 18 patients were considered to have entered the dilated phase of hypertrophic cardiomyopathy (dilated hypertrophic cardiomyopathy), based on the familial nature of the disease and echocardiographic findings of progressive left ventricular wall thinning and cavity dilatation. In a further four patients a presumptive diagnosis of dilated cardiomyopathy was made before transplantation or death; this diagnosis was then refined to that of dilated hypertrophic cardiomyopathy on the basis of the histological findings (more than $10 \%$ of the myocardium occupied by myocyte disarray). 
TISSUE PREPARATION

All hearts were fixed in $10 \%$ buffered formalin and weighed (body weight was available in only 18 cases and it was therefore not possible to adjust for body size). Full thickness tissue blocks were taken in a plane perpendicular to the long axis of the left ventricle at three levels: $1 \mathrm{~cm}$ below the aortic valve ring (base); at the papillary muscle tips (mid); and approximately $3 \mathrm{~cm}$ above the apex (apical). Trabeculations and papillary muscle were excluded from tissue blocks.

At each level, sections were obtained from the anterior and posterior septum, anterior wall, posterior wall, lateral left ventricular free wall, and right ventricle. In all, 18 sections of the left and right ventricles were obtained. Full thickness blocks of the left atrial free wall were taken in planes both perpendicular to and parallel to the long axis of the left ventricle.

All sections were embedded in paraffin, sectioned at $6 \mu \mathrm{m}$ thickness, and stained with haematoxylin and eosin, and elastic van Gieson.

MACROSCOPIC EVALUATION

The anterior-posterior left ventricular cavity diameter was measured at the mid-cavity level (which has been shown to correlate most closely with the left ventricular end systolic dimension measured at echocardiography ${ }^{13}$ ). Subaortic mitral valve impact lesions were noted. Both atria were examined and classified by visual inspection as normal or dilated.

The wall thickness was measured before sectioning. An anterior septum to posterior free wall ratio of $\geqslant 1.3$ was taken as evidence of asymmetrical septal hypertrophy. Distal left ventricular hypertrophy was defined as a wall thickness of more than $20 \mathrm{~mm}$ below the papillary muscle level. Left or right atrial wall hypertrophy, or both, was noted by visual inspection.

Fibrosis visible to the naked eye was graded 1 if absent, 2 if mild, and 3 if severe. Where present, the site of discrete replacement scars was noted and the size recorded as $<5 \mathrm{~mm}$ or $>5 \mathrm{~mm}$ in diameter.

Macroscopic evaluation was limited in some patients due to previous dissection or the nature of explanted hearts.

\section{MICROSCOPIC EVALUATION}

Definition and assessment of disarray

Cardiac myocyte arrangement was considered abnormal when type 1 a disorganisation was found (areas of myocardium in which adjacent myocytes are oriented either obliquely or perpendicularly to each other). ${ }^{14}$

Each of the 19 sections was examined field by field (avoiding overlap), and scored as disarray present or absent. The number of fields in which disarray was present was divided by the total number of fields examined for each section (approximately 60/section), and a percentage derived for the presence of disarray.

Assessment of small vessel disease

Analysis of small vessels was made only of those intramyocardial vessels that could be viewed in cross section. It was undertaken in six sections of the left ventricle and one section of the right ventricle at mid-cavity level.

The external diameter (bordered by adventitial tissue) and luminal diameter (bounded by the endothelium) were measured for all suitable intramyocardial vessels with the use of a digitised pad and the Vids-V program (AMS, Cambridge, UK).

Five control hearts (with hypertensive left ventricular hypertrophy) were also studied. In none of these controls was the ratio of external diameter to lumen diameter more than 2.5 for any of the small vessels assessed (approximately 20-30 vessels in each tissue section). Thus to allow small vessel dysplasia to be assessed as a continuous variable, the severity of disease was taken as the percentage of vessels within a given section in which the external diameter to lumen diameter ratio was $\geqslant 3.0$. In the atria, small vessel disease was assessed both quantitatively (as with the rest of the heart) and also by simple inspection.

\section{Quantification of fibrosis}

Sections in which fibrous tissue was stained with elastic van Gieson were examined using the Optomax automated system (AMS, Cambridge, UK), in which stained tissue was first calibrated for each slide and then quantitated as a percentage of fibrosis across each field.

\section{STATISTICAL ANALYSIS}

Data are expressed as mean (SD). Both the mean and maximum values across the 15 sections of the left ventricle were analysed. Statistical analysis was performed using the independent $t$ test, $\chi^{2}$ test, and correlation coefficient and multilinear regression analysis where appropriate. Within each heart, simple Pearson correlation testing was used to derive correlation coefficients between all of the following: wall thickness, disarray, fibrosis, and small vessel disease. The sign test was then used to assess whether the number of positive or negative correlation coefficients was significant. A probability value of $\mathrm{p}<0.05$ was considered significant.

\section{Results}

The mean age was 35.7 (17.8) years (range 6-81 years), with male patients comprising $59 \%$ of the total. Twenty two (30\%) were considered to have dilated hypertrophic cardiomyopathy, with a mean age significantly greater than the remainder (45.9 (16.9) years $v 30.6$ (16) years, $p=0.001$ ).

The distribution of gross and histological findings is summarised in table 1 .

GROSS FINDINGS

Heart weight

Mean heart weight was 529.6 (221.9) g (range $170-1550 \mathrm{~g}$ ) and varied according to sex, being significantly greater in male patients than in female patients $(589.2 \quad(240.5) \mathrm{g} v 446.7$ (159.4) g; p =0.006). There was no association between age and heart weight $(r=0.1$, $\mathrm{p}=0.5$ ), either for the group as a whole or in a separate analysis of those over 12 years of age. 
Table 1 The distribution of macroscopic and microscopic findings in hypertrophic cardiomyopathy

\begin{tabular}{|c|c|c|c|c|c|c|c|}
\hline & \multicolumn{5}{|l|}{ Sections of the heart } & \multirow{2}{*}{$\begin{array}{l}\text { Total } \\
\text { across all } \\
\text { sections of } \\
\text { the LV }\end{array}$} & \multirow[b]{2}{*}{ Right ventricle } \\
\hline & Anterior septum & Posterior septum & Anterior wall & Posterior wall & Lateral wall & & \\
\hline $\begin{array}{l}\text { Wall thickness }(\mathrm{mm}) \text { at } \\
\text { base (range) }\end{array}$ & $18.9(8-40)$ & $16.0(6-35)$ & $19.5(9-30)$ & $15.3(8-29)$ & $15.3(3.5-28)$ & 17.0 & $6.1(2-12)$ \\
\hline $\begin{array}{l}\text { Wall thickness (mm) at } \\
\text { mid level (range) }\end{array}$ & $20.2(2-37)$ & $19.8(5-35)$ & $18.0(2-34)$ & $14.8(5-27)$ & $16.7(7-28)$ & 17.9 & $6.4(2-15)$ \\
\hline $\begin{array}{l}\text { Wall thickness (mm) at } \\
\text { apex (range) } \\
\% \text { Disarray at base (range) }\end{array}$ & $\begin{array}{l}16.3(7-28) \\
26.7(0-100)\end{array}$ & $\begin{array}{l}16.1(5-27) \\
25.5(0-90.5)\end{array}$ & $\begin{array}{l}14.7(3-28) \\
24.5(0-68.7)\end{array}$ & $\begin{array}{l}14.9(5-28) \\
21.6(0-93)\end{array}$ & $\begin{array}{l}15.4(5-28) \\
15.55(0-74.2)\end{array}$ & $\begin{array}{l}15.5 \\
22.8\end{array}$ & $\begin{array}{l}5.9(2-15) \\
16.1(0-64)\end{array}$ \\
\hline $\begin{array}{l}\% \text { Disarray at mid } \\
\text { level (range) } \\
\% \text { Disarray at apex (range) } \\
\% \text { Fibrosis at base (range) }\end{array}$ & $\begin{array}{l}30.5(0-81) \\
22.4(0-65.8) \\
2.83(0.1-19.3)\end{array}$ & $\begin{array}{l}26.3(0-72.7) \\
24.8(0-80.3) \\
1.92(0.05-8.6)\end{array}$ & $\begin{array}{l}28.7(0-80.5) \\
21.4(0-78.7) \\
3.47(0.05-21.2)\end{array}$ & $\begin{array}{l}18.9(0-62.9) \\
22.1(0-69.2) \\
1.38(0.03-5.27)\end{array}$ & $\begin{array}{l}12.9(0-71.4) \\
16.8(0-74) \\
1.9(0.03-21.9)\end{array}$ & $\begin{array}{r}23.5 \\
21.5 \\
2.3\end{array}$ & $\begin{array}{l}17.1(0-72.5) \\
14(0-41.7) \\
1.06(0.02-7.7)\end{array}$ \\
\hline $\begin{array}{l}\% \text { Fibrosis at mid } \\
\text { level (range) } \\
\% \text { Fibrosis at apex (range) } \\
\% \text { Small vessel disease at } \\
\text { mid level (range) }\end{array}$ & $\begin{array}{l}2.25(0.1-23.8) \\
2.42(0.05-15.8) \\
15.1(0-53)\end{array}$ & $\begin{array}{l}2.8(0.1-16.6) \\
3.42(0.1-16.7) \\
13.7(0-57.4)\end{array}$ & $\begin{array}{l}3.3(0.1-18.5) \\
2.64(0.1-16.83) \\
15.9(0-64.2)\end{array}$ & $\begin{array}{l}2.3(0.04-15.1) \\
2.33(0.2-22.4) \\
14.5(0-47.6)\end{array}$ & $\begin{array}{l}1.7(0.03-21.9) \\
1.69(0.1-16.9) \\
11.6(0-39.5)\end{array}$ & $\begin{array}{r}2.5 \\
2.5 \\
14.2\end{array}$ & $\begin{array}{l}1.06(0.05-5.1) \\
1.77(0.09-23.4) \\
14.6(0-50)\end{array}$ \\
\hline
\end{tabular}

$\mathrm{LV}$, left ventricle.

However, all three patients with massive heart weights $(>900 \mathrm{~g}$ ) were under the age of 30 years.

Heart weight did not differ significantly according to whether the diagnosis was hypertrophic cardiomyopathy or dilated hypertrophic cardiomyopathy (505.5 (198.9) g v 589.4 (265.6) g; $\mathrm{p}=0.2$ ).

Distribution of left ventricular hypertrophy Maximum left ventricular wall thickness was $23.3(7.4) \mathrm{mm}$ (range $3.4-40 \mathrm{~mm}$ ). Asymmetrical septal hypertrophy was present in $65 \%$ of the patients, while distal left ventricular hypertrophy was noted in 19 of 64 patients $(30 \%)$. No cases of isolated distal or true apical hypertrophy were seen. One case of isolated subaortic septal hypertrophy was noted.

A right ventricular wall thickness of more than $4 \mathrm{~mm}$ was found in 41 of 64 patients $(64 \%)$, and $\geqslant 8 \mathrm{~mm}$ in $18(28 \%)$.

There was no correlation between maximum wall thickness and age $(r=-0.01, \mathrm{p}=0.9)$. However, patients aged $\leqslant 30$ years (but over 12 years) had a greater wall thickness than those over the age of 30 (maximum wall thickness of $26.5(8.7) \mathrm{mm} v 22.2(6.3) \mathrm{mm} ; \mathrm{p}=0.02)$.

Maximum left ventricular wall thickness was greater in male than in female patients (25.2 (7.4) $\mathrm{mm} v 20.3$ (6.4) $\mathrm{mm} ; \mathrm{p}=0.006)$.

Left or right atrial wall hypertrophy, or both, was noted in seven patients.

\section{Mitral valve impact lesion and left atrial dilatation}

A mitral valve impact lesion was visible in 29 of 65 hearts assessed (45\%), and left atrial dilatation was present in 28 of $62(45 \%)$. A mitral valve impact lesion was no more common in patients with dilated hypertrophic cardiomyopathy than in those with typical hypertrophic cardiomyopathy $(p=0.6)$, nor was it associated with a significantly greater heart weight (546.9 (161.4) g v 495.1 (251.6) g in patients without an impact lesion, $\mathrm{p}=0.3$ ). However, hearts with an impact lesion had a greater maximum left ventricular wall thickness (25.5 (6.8) $\mathrm{mm} v 21.9$ (7.3) mm, $\mathrm{p}=0.04)$.
Replacement scarring (macroscopic fibrosis)

The macroscopic fibrosis score was directly related to age at death $(r=0.2, \mathrm{p}=0.006)$, heart weight $(r=0.53, \mathrm{p}<0.001)$, and left ventricular cavity dimension $(r=0.4$, $\mathrm{p}=0.002$ ).

Of those in whom discrete scarring was assessed $(n=57), 32$ patients had small or no scars $(<5 \mathrm{~mm})$ and 25 had notable discrete scarring (5-10 $\mathrm{mm}$ in diameter).

Patients with little scarring differed from those with extensive scarring with respect to age (mean age 26.5 (15.9) years $v 33.5$ (4.9) years, respectively; $\mathrm{p}=0.25)$, and sex (19 $(76 \%)$ of the patients with large, discrete scars were male and six (24\%) were female; $\mathrm{p}=0.07)$.

\section{HISTOLOGICAL FINDINGS}

\section{Disarray}

Variation within a heart was great, such that the per cent disarray in one heart was seen to range from under $10 \%$ in one section to $90 \%$ in another. This variation was also seen within the same section, such that severely disarrayed myocytes could be seen to lie adjacent to normally sized and normally aligned myocytes (fig 1). Indeed this patchy distribution seemed characteristic of the disease in all hearts examined.

Disarray was less prominent in dilated hypertrophic cardiomyopathy than in typical

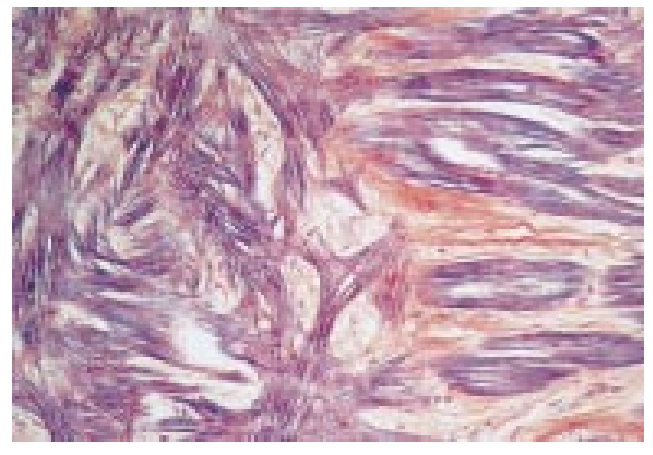

Figure 1 Section showing focal distribution of myocyte disarray (to the left) adjacent to normal parallel alignment of myocytes ( $\times 16$ objective). 
hypertrophic cardiomyopathy (mean disarray $17.2(11.5) \%$ v $28(18.8) \%, \mathrm{p}=0.004)$ (fig 2A).

Disarray was not significantly related to heart weight $(r=-0.1, \mathrm{p}=0.5)$, even after excluding patients with dilated hypertrophic cardiomyopathy. There was also no association between disarray and wall thickness $(r=-0.1$, $\mathrm{p}=0.3$ ) or pattern of left ventricular hypertrophy (mean disarray in patients with anterior septum to posterior free wall ratio $\geqslant 1.3$ was $23.4(15.4) \%$, v $23.9(17.4) \%$ for those $<1.3$, $\mathrm{p}=0.9$ ). However, after patients with dilated hypertrophic cardiomyopathy were excluded, disarray was significantly greater in hearts with maximum wall thickness of $\leqslant 20 \mathrm{~mm} v>20$ $\mathrm{mm}$ (60.9 (26)\% v 43 (20.4)\%, respectively; $\mathrm{p}=0.02)$. Within each heart the correlation coefficients for wall thickness versus disarray were positive in 52 hearts and negative in only 20; this was highly significant $(p=0.0002)$.

Disarray was greater in patients without a mitral valve impact lesion than in those who had an impact lesion (mean disarray 26.3 $(17.7) \%$ v $18.9 \quad(11.6) \%$, respectively; $\mathrm{p}=0.04)$.

Pronounced disarray was present in the very young (up to $80 \%$ of the myocardium was occupied by myocyte disarray in a six year old patient) and mean per cent disarray was inversely related to age $(r=-0.44, \mathrm{p}<0.001)$. This relation was confirmed when patients were grouped according to age $<30$ years $v$ $\geqslant 30$ years (mean disarray of $29(20.2) \% v$ $19.9(14) \%, p=0.04)$. There was no significant difference in the severity of disarray
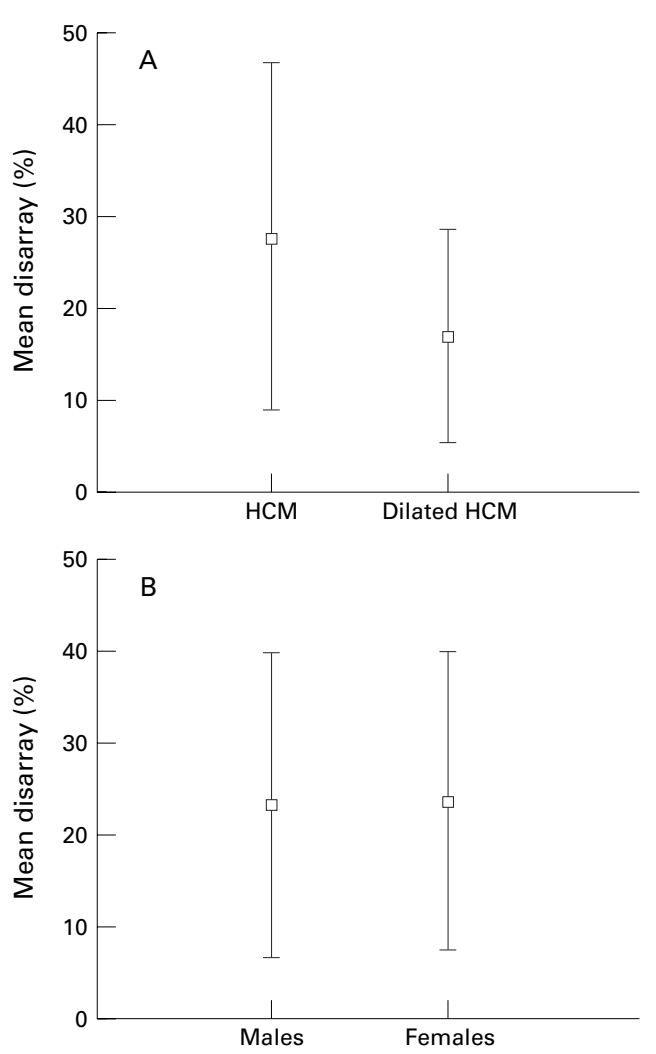

Figure 2 Disarray v disease status $(A)$ and $\operatorname{sex}(B)$. between male and female patients (mean disarray $23.5(16.7) \% v 25.8(19) \%, p=0.6)$ (fig 2B).

No disarray was seen in any section of the left atrial free wall.

\section{Fibrosis}

The distribution of fibrosis is shown in table 1 .

A weak but highly significant relation was seen between fibrosis and heart weight $(r=0.4, \mathrm{p}=0.001)$, and between fibrosis and maximum left ventricular wall thickness $(r=0.3, \mathrm{p}=0.03)$, and within each heart the correlation coefficients for wall thickness versus fibrosis were positive in 48 and negative in 24 cases $(\mathrm{p}=0.006)$.

Microscopic fibrosis was greater in hearts with dilated hypertrophic cardiomyopathy than in those with hypertrophic cardiomyopathy (9.2 (7.3)\% $v 4.1(4.9) \%, \mathrm{p}=0.001)$, and in male versus female patients $(7 \quad(6.9) \%$ v 4 (4.6) $\%, p=0.04$ ) (fig 3A,B), while per cent fibrosis showed a direct relation to age $(r=0.4$, $\mathrm{p}=0.03)$.

The severity of atrial fibrosis did not differ significantly in patients with or without left atrial dilatation $(0.9(0.9) \% \quad v \quad 1.03(0.6) \%$, $\mathrm{p}=0.7)$, or in patients with or without a mitral valve impact lesion $(0.9(0.8) \% v 1.05(0.9) \%$, $\mathrm{p}=0.6)$.

\section{Small vessel disease}

There was no significant relation between small vessel disease and age $(r=0.3, \mathrm{p}=0.3)$ or sex (maximum small vessel disease 26.7 (16.4)\% in male patients $v 23.8$ (11.9)\% in female
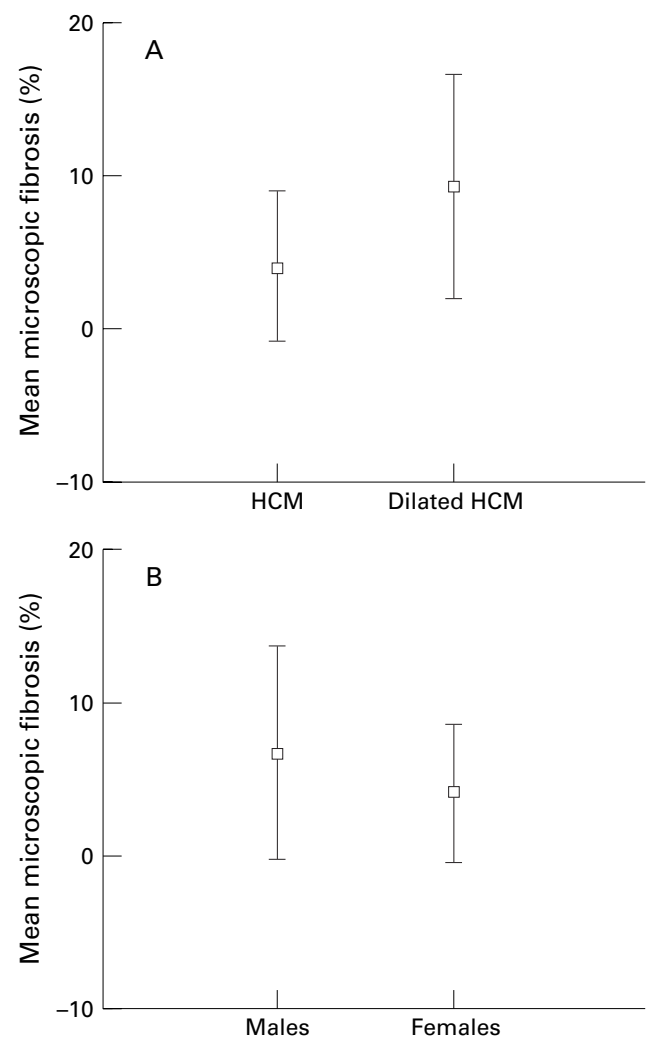

Figure 3 Microscopic fibrosis versus disease status (A) and $\operatorname{sex}(B)$. 
patients, $p=0.4$ ). A weak but significant correlation was found between small vessel disease and heart weight $(r=0.3, \mathrm{p}=0.01)$, and between small vessel disease and maximum left ventricular wall thickness $(r=0.3$, $\mathrm{p}=0.03$ ). However, a poor correlation was seen between hypertrophy and small vessel disease on analysing the data within individual hearts. This latter finding may, however, reflect the fact that small vessel disease was examined in too few segments to achieve significance (seven segments $v 19$ segments in which disarray and fibrosis were studied).

No small vessel disease was seen in the atria of 29 patients.

Small vessel disease was found in three of four patients aged $\leqslant 12$ years (the youngest was 6 years old); although it appeared much less severe, the difference did not reach significance (maximum small vessel disease 13.6 (11.7)\% (range 0-28.6\%) v 26 (14.8)\% (range $0-64.3 \%$ ) in patients over 12 years of age, $\mathrm{p}=0.1)$.

\section{Interrelations of histological features}

No significant correlation was found between disarray and fibrosis $(r=-0.5, \mathrm{p}=0.6)$, disarray and small vessel disease $(r=-0.2, \mathrm{p}=0.1)$, or fibrosis and small vessel disease $(r=0.8$, $\mathrm{p}=0.5)$. These analyses were performed in the total patient population and in "typical" hypertrophic cardiomyopathy patients only (that is, after exclusion of patients with dilated hypertrophic cardiomyopathy). Analysing fibrosis, disarray, and small vessel disease within individual hearts confirmed this poor correlation.

In some sections abnormal intramural small arteries were present in association with fibrosis; however, severely dysplastic small vessels

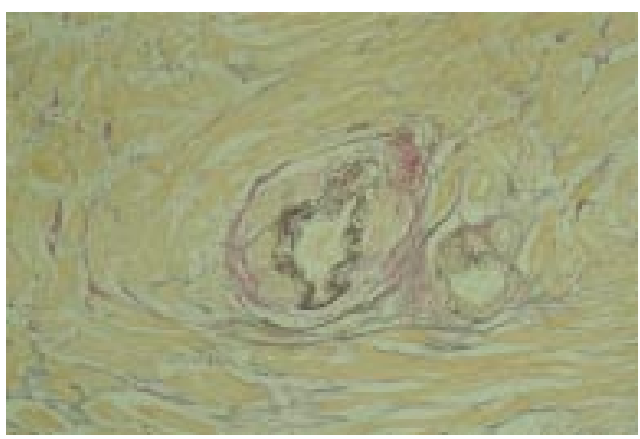

Figure 4 Dysplastic small vessel with no surrounding microscopic fibrosis ( $\times 10$ objective).

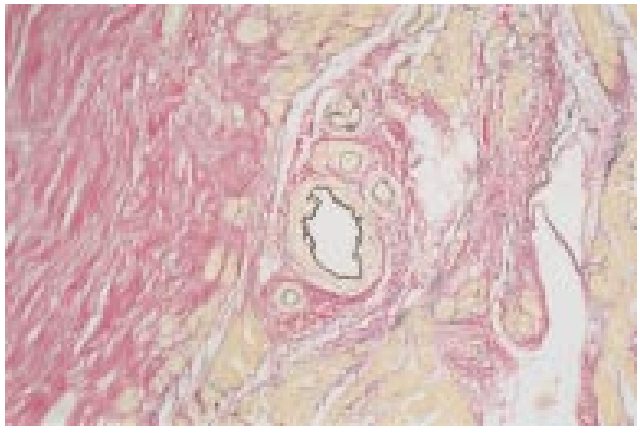

Figure 5 Pronounced fibrosis (sheets of collagen stained pink) with adjacent normal small vessel $(\times 10$ objective). were also seen in areas without pronounced fibrosis, while sections with widespread fibrosis often contained normal small vessels (figs 4 and 5).

\section{Discussion}

We set out to assess in detail the macroscopic and histological features of a large group of patients with hypertrophic cardiomyopathy. To ensure a comprehensive survey we elected to include patients with normal heart weights and widespread disarray and those with dilated hypertrophic cardiomyopathy, as both of these phenotypes are recognised as a part of the hypertrophic cardiomyopathy spectrum. Patients with dilated hypertrophic cardiomyopathy had significantly less disarray, probably as a result of myocyte loss and replacement fibrosis (hence areas of disarray are replaced with fibrous tissue). Alternatively these patients may have a distinct phenotype from the outset with much less disarray and an increased risk of fibrosis and wall thinning.

Disarray was also significantly greater in patients without a mitral valve impact lesion, supporting the results of an earlier study by Maron and colleagues, ${ }^{8}$ who found the overall disarray score to be greater in the group with no left ventricular outflow tract obstruction on echocardiography. In addition, the presence of an impact lesion was found to be independent of heart weight, suggesting that systolic anterior motion of the mitral valve (which is the probable cause of an impact lesion) is gene specific and does not arise as a direct response to pronounced hypertrophy.

In a transgenic animal model of hypertrophic cardiomyopathy, male mice were found to have much worse histology. ${ }^{15}$ Furthermore, transgenic mice with hypertrophic cardiomyopathy showed myocyte disarray as an early response to the mutated genotype that preceded the onset of both hypertrophy and fibrosis..$^{15}$ In our study, a sex specific influence was seen with respect to heart weight, wall thickness, fibrosis, and scarring, with male patients showing a more severe phenotype. However, sex had no influence on disarray. It would thus appear that, for some features at least, male patients are at greater risk. Furthermore, fibrosis increased with age, while disarray was present at an early age and diminished with time. These results suggest that in human disease, as in the mouse model, disarray is a primary response to the mutated gene, while fibrosis and hypertrophy are later, secondary responses.

Young patients had a greater maximum left ventricular wall thickness, supporting an earlier clinical study in which left ventricular hypertrophy on echocardiography was considerably more severe in the young. ${ }^{16}$ In addition, all of our patients with massive heart weights were under the age of 30 years, suggesting that massive left ventricular hypertrophy is indeed a risk factor for premature death. As fibrosis but not disarray increases with increasing heart weight, it is likely that the mechanism of sudden death in these patients is related to marked fibrosis and scarring, rather than to disarray. 
Age was also found to have an inverse relation with disarray, suggesting an increased risk of premature sudden cardiac death in patients with extensive disarray.

Analysis of the patient group as a whole showed no correlation between the degree of disarray and heart weight or maximum left ventricular wall thickness. This has been reported in previous studies, which also included patients with sudden death and cardiac failure. ${ }^{14}$ However, after excluding patients with dilated hypertrophic cardiomyopathy it was seen that disarray was significantly greater in patients with only mild left ventricular hypertrophy (wall thickness $<20 \mathrm{~mm}$ ). This provides further evidence that hypertrophy is not a response to myocyte disarray (nor indeed is disarray a response to pronounced hypertrophy).

We found great variability in the distribution of disorganised myocytes, not only from heart to heart but also within each heart. From what we know so far of the molecular biology of hypertrophic cardiomyopathy it is not easy to explain this very patchy appearance. It might reflect differential gene expression-for example, as a result of random methylation of the diseased gene or mitochondrial DNA heteroplasmy. Cell to cell differences in autocrine production of factors such as transforming growth factor $\beta$ or tumour necrosis factor may also play a role.

Despite the frequency of atrial abnormalities in hypertrophic cardiomyopathy (both atrial dilatation and atrial arrhythmias), the histology of atrial tissue has not been reported in the human disease. None of the hearts examined showed any evidence of myocyte disarray within atrial tissue, and it is difficult to find an easy explanation for this. It is unlikely to reflect problems of assessing myocyte arrangement at this site as the rather irregularly aligned cell arrangement would in general lead to an overestimation rather than to an underestimation of disarray. Equally, it seems unlikely that the absence of disarray in the atria results from haemodynamic factors, as left atrial pressures in hypertrophic cardiomyopathy may reach those of the right ventricle where disarray is seen. Alternatively, it is possible that the atrial tissue examined may have been taken from only those hearts in which the mutated protein is not expressed in the atria-for example, the $\beta$ myosin heavy chain protein is not the predominant isoform in human atrial tissue. This issue can only be addressed by the genotyping of necropsy samples. However, it seems unlikely that a heart with, for example, a troponin $\mathrm{T}$ mutation (the protein product being expressed in atrial tissue) was not included, because of the large number of samples examined. Instead, it could be postulated that local autocrine factors might be responsible for modifying gene expression in the atria, or that sarcomeric proteins unique to the ventricles are necessary for disease expression.

It is of interest that small vessel disease was not related directly to age and was present in the very young. Maron and colleagues reported the histology of a young patient with hypertrophic cardiomyopathy presenting with sudden death and also found a notable degree of small vessel abnormality within the myocardium ${ }^{17}$; in a separate study they observed small vessel disease in three of eight infants with hypertrophic cardiomyopathy (these changes were not associated with fibrosis). ${ }^{9}$ Taken together, these results suggest that small vessel disease is an early feature of hypertrophic cardiomyopathy. However, it is possible that the small vessel disease seen in these very young patients is the basis for their sudden and extremely premature deaths. All hearts examined in this age range were acquired after sudden death, and it is therefore not possible to determine whether in most children with hypertrophic cardiomyopathy small vessel disease does not generally develop until much later.

A feature of our study was the quantitative nature of small vessel disease assessment. All previous studies in patients with hypertrophic cardiomyopathy have assessed small vessel disease in at best a semiquantitative manner, stating whether abnormalities of the small vessels were present or not. ${ }^{9}$ This method is obviously prone to errors of subjectivity, and the bias of associating abnormal small vessels with areas of fibrosis may be reinforced. Interestingly, we found no relation between small vessel disease and fibrosis in our patients, which would seem to conflict with previous reports. ${ }^{9}{ }^{18}$ However, we cannot rule out an association between a specific subtype of myocardial fibrosis and small vessel disease, as our methods did not discriminate between, for example, plexiform and perivascular fibrosis. It may well be, therefore, that the total fibrosis content of the myocardium is unrelated to small vessel disease, but that a specific form of collagen deposition is associated with small vessel changes.

Interestingly, at each section within an individual heart both disarray and fibrosis correlated with the distribution of hypertrophy. However, in the overall population maximum wall thickness correlated with fibrosis but not with disarray. Instead, mild left ventricular hypertrophy (maximum wall thickness $<20 \mathrm{~mm}$ ) was associated with greater disarray in patients with preserved systolic function. This suggests that the maximum wall thickness does not predict the degree of disarray, but that within each heart the distribution of hypertrophy does reflect the distribution of histological features.

\section{STUDY LIMITATIONS}

The hearts examined were obtained after death or after cardiac transplantation. This may have biased the results towards examination of a more severe phenotype, and the results may therefore not be readily applicable to all patients with this disease.

\section{CONCLUSION}

We suggest that myocyte disarray is a direct response to functional or structural abnormalities of the mutated sarcomeric protein, while fibrosis and small vessel disease are secondary phenomena unrelated to disarray, but modified by factors such as left ventricular mass, sex, and perhaps local autocrine factors. 
AMV is supported by a clinical training fellowship from the Medical Research Council. SS is supported by a junior research fellow grant from the British Heart Foundation. We would like to thank Annie O'Donoghue and Jan Poloniecki for their assistance.

1 Teare D. Asymmetrical hypertrophy of the heart in young adults. Br Heart f 1958;20:1-8.

2 Davies MJ. The current status of myocardial disarray in hypertrophic cardiomyopathy. Br Heart f 1984;51:361-3.

$3 \mathrm{St}$ John Sutton MG, Lie JT, Anderson KR, et al. Histopathological specificity of hypertrophic obstructive cardiomyopathy. Myocardial fibre disarray and myocardial fibrosis. Br Heart $\mathcal{F}$ 1980;44:433-43.

4 Maron BJ, Wolfson JK, Epstein SE, et al. Morphologic evidence for "small vessel disease" in patients with hypertrophic cardiomyopathy. Z Kardiol 1987;76(suppl 3):91100 .

5 Marian AJ, Roberts R. Recent advances in the molecular genetics of hypertrophic cardiomyopathy. Circulation 1995; 92:1336-47.

6 Schwartz K, Carrier L, Guicheney P, et al. Molecular basis of familial cardiomyopathies. Circulation 1995;91:532-40.

Watkins H. Genotype:phenotype correlations in hypertrophic cardiomyopathy. Eur Heart f 1998;19:10-12.

8 Maron BJ, Ferrans VJ, Henry WL, et al. Differences in distribution of myocardial abnormalities in patients with tribution of myocardial abnormalities in patients with
obstructive and nonobstructive asymmetric septal hyperobstructive and nonobstructive asymmetric septal hyper-
trophy (ASH). Light and electron microscopic findings.

9 Maron BJ, Wolfson JK, Epstein SE, et al. Intramural ("small vessel") coronary artery disease in hypertrophic cardiomyopathy. $\mathcal{F}$ Am Coll Cardiol 1986;8:545-57.
10 ten Cate FJ, Roelandt J. Progression to left ventricular dilatation in patients with hypertrophic obstructive cardiomyopathy. Am Heart f 1979;97:762-5.

11 Yutani C, Imakita M, Ishibashi-Ueda $\mathrm{H}$, et al.. Three autopsy cases of progression to left ventricular dilatation in patients with hypertrophic cardiomyopathy. Am Heart $\mathcal{f}$ 1985;109:545-53.

12 Maron BJ, Roberts WC. Hypertrophic cardiomyopathy and cardiac muscle cell disorganization revisited: relation between the two and significance. Am Heart $\mathcal{F}$ 1981;102: 95-110.

13 Maron BJ, Henry WL, Roberts R, et al. Comparison of echocardiographic and necropsy measurements of ventricular wall thicknesses in patients with and without disproportionate septal thickening. Circulation 1977;55: disprop.

14 Maron BJ, Wolfson JK, Roberts WC. Relation between extent of cardiac muscle cell disorganisation and left ventricular wall thickness in hypertrophic cardiomyopathy. Am 7 Cardiol 1992;70:785-90.

15 Geisterfer-Lowrance AA, Christe M, Conner DA, et al. A mouse model of familial hypertrophic cardiomyopathy. Science 1996;272:731-4.

16 Spirito P, Maron BJ. Relation between extent of left ventricular hypertrophy and age in hypertrophic cardiomycontrio $\mathrm{Am}$ Coll Cardiol 1989;13:820-3.

17 Maron BJ, Kragel AH, Roberts WC. Sudden death in hypertrophic cardiomyopathy with normal left ventricular hypertrophic cardiomyopathy with
mass. Br Heart $\mathcal{F}$ 1990;63:308-10.

18 Tanaka M, Fujiwara H, Onodera T, et al. Quantitative analysis of narrowings of intramyocardial small arteries in normal hearts, hypertensive hearts, and hearts with hypertrophic cardiomyopathy. Circulation 1987;75:1130-9.

\section{IMAGES IN CARDIOLOGY}

\section{Visualisation of systolic myocardial perfusion defect induced by septal squeezing}
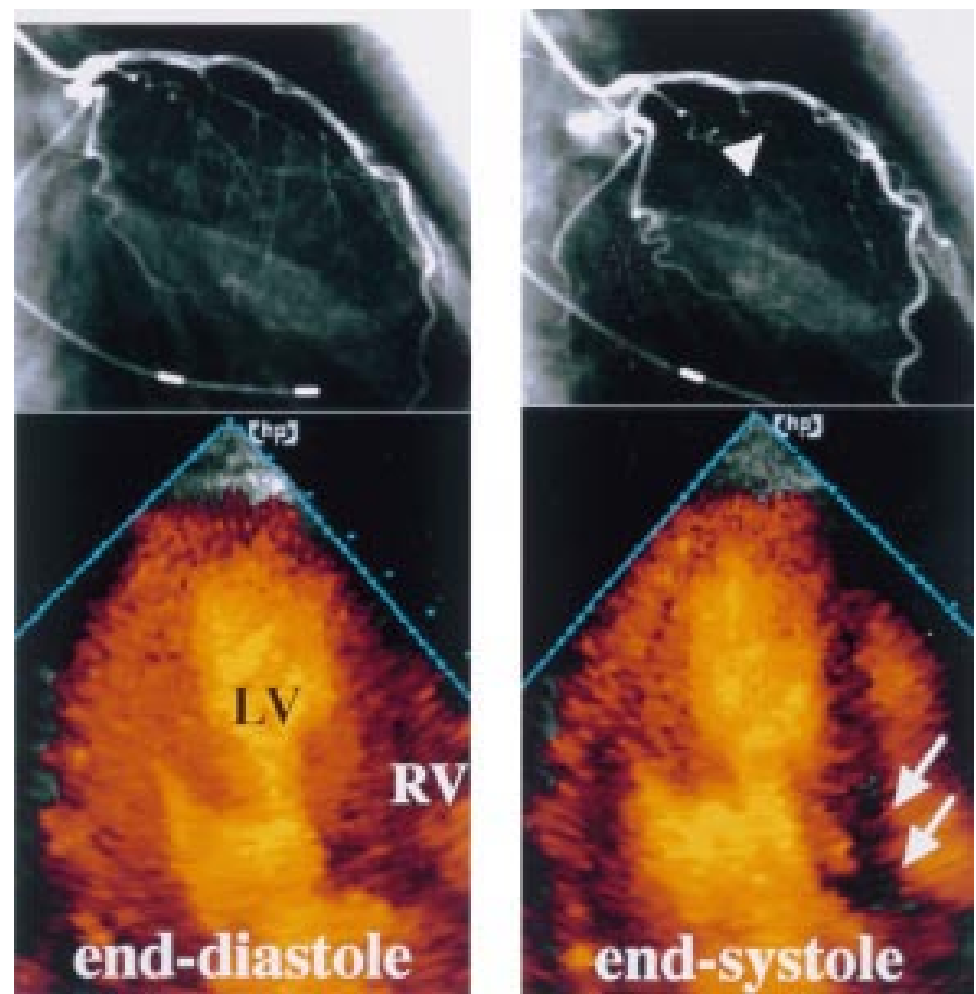

A 58 year old woman presented with exertional dyspnoea and chest pain. Cross sectional and Doppler echocardiography revealed asymmetric septal hypertrophy, systolic anterior movement of the anterior mitral leaflet, and left ventricular outflow obstruction with a pressure gradient of $108 \mathrm{~mm} \mathrm{Hg}$. The patient underwent right and left heart catheterisation, including right ventricular endocardial biopsies, and was diagnosed with hypertrophic obstructive cardiomyopathy. Coronary arteriography revealed prominent systolic myocardial squeezing of the septal branches of the left anterior descending artery with no significant arterosclerotic stenosis (top, arrowheads). We performed myocardial contrast echocardiography with ECG triggered harmonic Doppler technique using intravenous Levovist (a saccharide based transpulmonary echo contrast agent; Schering AG). The image triggered at end diastole showed homogeneous myocardial opacification (bottom, left). In contrast, the end systolic image showed a myocardial perfusion defect at the proximal septum which was consistent with coronary arterial squeezing (bottom, right). This unusual case illustrates the use of myocardial contrast echocardiography to identify the difference in coronary perfusion between diastole and systole. 